\title{
Relationship of oscillating and average components of laser Doppler flowmetry signal
}

\author{
Irina Mizeva \\ Peter Frick \\ Sergey Podtaev
}




\title{
Relationship of oscillating and average components of laser Doppler flowmetry signal
}

\author{
Irina Mizeva, ${ }^{*}$ Peter Frick, and Sergey Podtaev \\ Institute of Continuous Media Mechanics, ak Koroleva 1, Perm 614013, Russia
}

\begin{abstract}
Signals from laser Doppler flowmeters widely used in intravital studies of skin blood flow include, along with a slowly varying average component, an oscillating part. However, in most clinical studies, pulsations are usually smoothed by data preprocessing and only the mean blood flow is analyzed. To reveal the relationship between average and oscillating perfusion components measured by a laser Doppler flowmeter, we examined the microvascular response to the contralateral cold pressor test recorded at two different sites of the hand: dorsal part of the arm and finger pad. Such a protocol makes it possible to provide a wide range of perfusion. The average perfusion always decreases during cooling, while the oscillating component demonstrates a differently directed response. The wavelet analysis of laser Doppler flowmetry (LDF) signals shows that the pulsatile component is nonlinearly related to the average perfusion. Under low perfusion, the amplitude of pulsations is proportional to its mean value, but, as perfusion increases, the amplitude of pulsations becomes lower. The type of response is defined by the basal perfusion and the degree of vasoconstriction caused by cooling. Interpretation of the results is complicated by the nonlinear transfer function of the LDF device, the contribution of which is studied using artificial examples. @ 2016 Society of Photo-Optical Instrumentation Engineers (SPIE) [DOI: 10.1117/1.JBO.21 .8.085002]
\end{abstract}

Keywords: skin blood flow oscillations; laser Doppler flowmetry; wavelet analysis; cold pressor test.

Paper 160088RR received Feb. 19, 2016; accepted for publication Jul. 18, 2016; published online Aug. 22, 2016.

\section{Introduction}

Blood flow pulsations in the microvessels have recently attracted significant interest in biomedical research ${ }^{1,2}$ and provide a basis for new diagnostic methods to be developed for clinical practice. ${ }^{3}$ Microcirculation is affected by nerve, humoral, and local factors, which influence both the average and oscillating components of the blood flow. ${ }^{4}$ However, in the majority of clinical studies, the pulsations are typically smoothed by data preprocessing and only the mean blood flow is taken into consideration. ${ }^{5}$ Below, we discuss this slowly varying component, which was obtained by the moving average filter as the average laser Doppler flowmetry (LDF) signal.

LDF, introduced in Ref. 6, has become an attractive technique for the intravital studies of cutaneous blood flow. It enables simple, real-time monitoring of the relative changes of red blood cell (RBC) flux in cutaneous microvascular bed, which consists of nutritive capillaries and deeper elements of the skin vascular tree. The individual difference in skin thickness and in the properties of the density of capillaries has a significant input in the variability of the LDF signals from person to person. Spontaneous oscillations of blood flux, observed by the LDF technique in rest conditions, have been demonstrated in a number of works. ${ }^{7,8}$

A common approach for testing the function of microcirculation is to perform functional tests inducing local or systemic changes in the skin blood flow. Usually, various stimuli are used, both pharmacological and physiological (i.e., occlusion, temperature, and mental). ${ }^{9}$ A massive stimulation of thermoreceptors during exposure to cold [i.e., the cold pressor test (CPT)] leads to activation of the sympathetic tone and a moderate increase of catecholamines in the blood plasma, but does not influence the frequency of the heartbeat. However, it must be noted that these processes may cause vasoconstriction (in arteries, arterioles, and arteriovenous anastomoses), which provokes a redistribution of blood in the microcirculation system. ${ }^{10,11}$

In a number of studies, vasoconstriction during the CPT is described, ${ }^{10,12}$ although less attention is devoted to the pulsations of blood flow. Recently, we found two types of reaction of LDF pulsations on the CPT, ${ }^{13,14}$ observing that in some cases CPT provokes an increase in the amplitude of pulsations, while in others, it leads to the opposite reaction. Assuming that a relationship between the mean value and oscillatory component of perfusion should exist (at least for cardiac pulsations), we then studied the microvascular oscillatory reaction in different frequency ranges in detail.

Usually, cutaneous blood flow is studied on the forearm, where capillary density is rather low, which allows to neglect the nonlinear dependence of LDF output $P_{\mathrm{LDF}}$ on the RBC concentration. In the present work, aiming to study the reaction of the average and pulsatile components of the LDF signal during the CPT to reveal their relationship, we consider maximum available range of perfusion, which is possible to reach on the palm surface of distal phalange.

\section{Background}

\subsection{Laser Doppler Flowmetry}

The LDF technique provides an index of skin perfusion by measuring the Doppler shift of coherent monochromatic light scattering by moving RBCs. The light penetrates to a depth 
of $\sim 1.5$ to $2 \mathrm{~mm}$ and the LDF signal corresponds to the mean blood flux in the volume near $1 \mathrm{~mm}^{3}$. It should be noted that a number of microvascular structures input in the LDF signal. The epidermis thickness is close to $0.4 \mathrm{~mm}$ and has no input in the LDF signal. Capillaries, which are in the surface of the dermis, are normally oriented to the surface. Deeper superficial plexus occur, under which communicating vessels (including venules, arterioles, and aortovenular shunts) are located. ${ }^{15}$

A laser Doppler instrument output provides the signal, which is quantified as the product of the average $\mathrm{RBC}$ velocity and concentration. ${ }^{16}$ This method utilizes the Doppler shift that occurs when light is scattered by moving RBCs. By analyzing the temporal fluctuations found in the speckle patterns formed by backscattered light from a laser illumination, Doppler shift can be detected indirectly. ${ }^{17} P_{\mathrm{LDF}}$ is linear to velocity of moving $\mathrm{RBC}$, but its relation with RBC concentration is more complicated. While RBC concentration is low, $P_{\mathrm{LDF}}$ is proportional to $\mathrm{RBC}$ concentration and saturates when the RBC concentration is high. ${ }^{18-20}$ This saturation of $P_{\mathrm{LDF}}$ can cause the reduction of oscillations amplitude at high RBC concentration. Subjects have individual variability of skin optic and microcirculation structure parameters and this fact does not allow to define boundary of the linear part of the transfer function. Mathematical modeling of backscattered radiation recorded by LDF showed that variability of perfusion can cause essential decrease of the signal to noise ratio. ${ }^{21}$ All these reasons give rise to the problem of nonlinear transfer function of LDF and the relation of LDF signal with the real blood flux. LDF does not provide an exact measure of flow, so it is often referred to as flux of the moving blood cells. The LDF output signal is in perfusion units (p.u.), which are relative and this causes some problems in data interpretation.

\subsection{Blood Flow Oscillation}

The blood microcirculation system is an active part of the cardiovascular system, where exchange processes between blood and tissue take place. The vascular wall, affected by nerve, humoral, and local factors, regulates the flow through the microcirculation system. ${ }^{15}$ These factors influence not only the mean flux rate through the microvascular system but also the oscillations of the blood flow. ${ }^{2,14}$ Mainly, oscillations are provided by the arterioles, which have walls that consist of smooth muscles. Arterioles receive autonomic nervous system innervations and respond to various circulating hormones to regulate their diameter. Changes in vasodilation and vasoconstriction conditions provide low-frequency pulsations of blood vessels that affect the luminal diameter, known as vasomotions. ${ }^{22}$ Vasomotions give rise to blood flow oscillations. ${ }^{23}$ This phenomena was described by Jones in 1852 and has since been reported in many vascular beds both in vivo and in vitro. It is extremely difficult to observe vasomotions in vivo and can only be done by means of intravital microscopy, which is difficult to apply. On the contrary, blood flow oscillations are easier to access by means of LDF measurements and some other methods, such as oxygen tension, local blood pressure measurements, and so on. ${ }^{24}$ Measured in vivo flow motions represent an integration of several periodic activities of different origin. ${ }^{2}$ In blood flow, oscillation mechanisms connected with central hemodynamic [cardiac $(0.8$ to $1.4 \mathrm{~Hz})$ and respiratory $(0.2$ to $0.4 \mathrm{~Hz})$ rates] are taken into consideration. Also, blood flow in microvessels is regulated by NO dependent endothelial activity $(0.01$ to $0.02 \mathrm{~Hz})$, myogenic activity
$(0.05$ to $0.14 \mathrm{~Hz})$, and by sympathetic nerves $(0.02$ to $0.05 \mathrm{~Hz}){ }^{1,7}$

The main role in the regulation of the number of functioning capillaries is played by the distributing microvessels, a system of meta-arteriols and precapillary sphincters. The diameter of the precapillary sphincters includes both static and dynamic components determined by the vasomotions. Their balance is important for the determination of the final hemodynamic effect on the capillary blood flow.

\subsection{Cold Pressure Test}

The contralateral cold pressure test excludes a direct influence on the vessels of the hand and indirectly provides strong vasoconstriction, ${ }^{25}$ which was first described by Lewis in $1930 .{ }^{26}$ Cutaneous receptors stimulated by the ice-water immersion of one hand increase sympathetic nerve activity to the palm skin in the nonimmersed contralateral hand and reduce the blood flow, which is reflected as a decrease in skin surface temperature ${ }^{27}$ under a constant ambient environment. ${ }^{11}$

The cutaneous circulation is a major effector of human thermoregulation, whereby, during cold stress, reduced temperatures lead to cutaneous vasoconstriction through combined neural and local mechanisms. ${ }^{11,14,28}$ Cold stress, during which the skin temperature decreases, initiates a thermoregulatory reflex to conserve body heat. This reflex is mediated by an increased noradrenergic vasoconstrictor tone, which causes an arteriolar vasoconstriction and a decrease in skin blood flow. ${ }^{25}$

\section{Subjects, Measurements, Mathematical Methods, and Software}

\subsection{Subjects}

LDF measurements were carried out for 14 healthy volunteers (six male and eight female) under controlled temperature $\left(24^{\circ} \mathrm{C}\right)$ conditions. There were no smokers or ex-smokers in the group, none of them received any medicine, and coffee was excluded at least for $4 \mathrm{~h}$ before the study. The mean age of the subjects was $35 \pm 5$. The subjects were sitting in a relaxed position. All the subjects gave their written informed consent prior to the test. The Ethical Committee of the Perm State Medical University approved the study protocol, and, in all cases, informed consent was received from the patients prior to the test.

\subsection{Measurements}

LDF was measured using a laser Doppler monitor (Moor Instruments FloLAB Server, U.K.) with a near-infrared laser (wavelength $780 \mathrm{~nm}$ ) and probe optical surface (MoorInstruments, VP-1 probe) attached to the tissue pulp of the index finger. In five subjects from the whole group, we simultaneously collected data from the palm surface of distal phalange and from the dorsal part of the palm between the base of the thumb and index finger (Fig. 1). The probe design minimized interference from external light sources. A bandwidth of $22 \mathrm{kHz}$ was used, which provided for a wideband measurement of RBC velocities, with easily discernible vasoconstrictor responses and minimal damping of the pulsatile component of the LDF trace. The sampling frequency was $40 \mathrm{~Hz}$. LDF probes were attached to the skin surface with double-sided adhesive disks, which avoided the disturbance of the blood supply of the skin that would have arisen of using a clamp. The perfusion $P$ was measured in arbitrary p.u. 


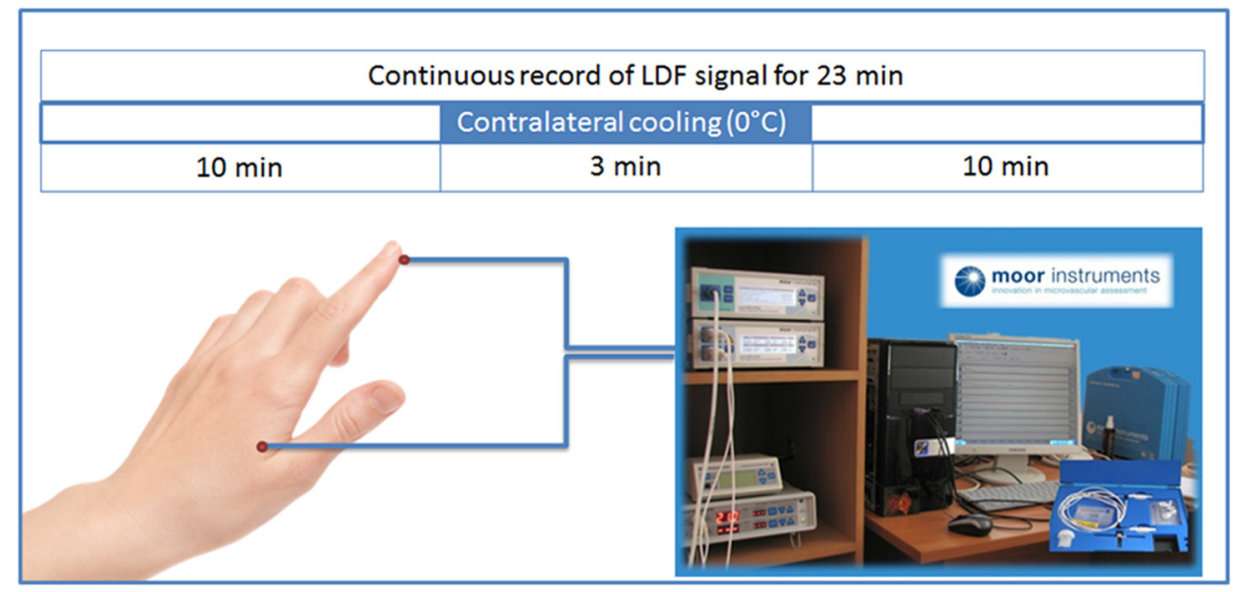

Fig. 1 Sketch of the experiment.

The CPT protocol was as follows: all the subjects were given 10 -min acclimatization period in the laboratory. The duration of signal collecting was $23 \mathrm{~min}$. In the first $10 \mathrm{~min}$, we recorded basal data from the left hand while the subject was in a relaxed condition. In the next $3 \mathrm{~min}$, the right (contralateral) hand was immersed in the bath with an ice-water mixture and then the right hand was put on the towel. This protocol design allowed us to minimize the moving artifacts, which could otherwise significantly affect the LDF signal. For the further analysis, we consider these three intervals before cooling (1 to $10 \mathrm{~min}$ ), the time interval when the cooling takes place (10 to $13 \mathrm{~min}$ ), and the recovery time after the cooling period (13 to $26 \mathrm{~min}$ ).

\subsection{Wavelet Analysis of Laser Doppler Flowmetry Data Series}

Accurate treatment of the frequency content of a nonstationary signal requires adequate mathematical instrumentation. For the spectral analysis and filtration, we used the continuous wavelet transform, an effective tool for analyzing short or nonstationary signals. ${ }^{29}$ The direct wavelet transform maps the recorded signal $f(t)$ into the time-frequency plane as

$W(\nu, \tau)=\nu \int_{-\infty}^{\infty} f(t) \psi^{*}[\nu(t-\tau)] \mathrm{d} t$,

where * means the complex conjugation. We used below one of the most popular wavelets, the Morlet wavelet, written in the form

$\psi(t)=e^{2 \pi i t} e^{-t^{2} / 2}$.

Then, for the spectral filtration, we made the inverse wavelet transform (IWT) into the corresponding frequency band

$f_{k}(t)=\frac{1}{C_{\psi}} \int_{\nu_{1}}^{\nu_{2}} \int_{-\infty}^{\infty} W(\nu, \tau) \psi[\nu(t-\tau)] \nu^{2} \mathrm{~d} \nu \mathrm{d} \tau$,

where $k$ indicates the frequency band from $\nu_{1}$ to $\nu_{2}$

$C_{\psi}=\frac{1}{2 \pi} \int_{-\infty}^{\infty} \frac{|\hat{\psi}(\nu)|}{|\nu|} \mathrm{d} \nu$

and $\hat{\psi}(\nu)$ is the Fourier transform of the analyzing wavelet.
The energy of pulsations in the corresponding frequency band is then

$M_{k}=\frac{1}{\left(t_{2}-t_{1}\right)} \int_{t_{1}}^{t_{2}} \int_{\nu_{1}}^{\nu_{2}}|W(\nu, t)|^{2} \mathrm{~d} t \mathrm{~d} \nu$.

As an estimation of the intensity of pulsations (amplitude) in the defined frequency band, the standard deviation (SD) of filtered signal at a given time interval can be used. However, neither the energy $M_{k}$ nor the corresponding SD allows tracing changes in the amplitude of pulsations via time or estimating the error of the calculated value. To avoid these disadvantages, we used another characteristic, first introduced in Ref. 30

$\tilde{f}_{k}(t)=\frac{1}{C_{\psi}} \int_{\nu_{1}}^{\nu_{2}}|w(\nu, \tau)| \nu \mathrm{d} \nu$,

which gives an envelope of $f_{k}(t)$. In Fig. 3 wavelet coefficients envelope is shown by red thick lines. $\tilde{f}_{k}(t)$ is a smoothed current characteristic of oscillation amplitude in the given frequency band, following Ref. 30, we will call it wavelet coefficient envelope (WCE).

Frequency-by-frequency cross-correlation analysis of the two signals $f(t)$ and $g(t)$ gives the wavelet cross-correlation function, which is a normalized wavelet cross-spectrum (see Ref. 31 for detailed description) used in the present paper

$C_{f g}(\nu)=\frac{\int_{t_{1}}^{t_{2}} W_{f}(\nu, \tau) W_{g}^{*}(\nu, \tau) \mathrm{d} \tau}{\sqrt{\int_{t_{1}}^{t_{2}}\left|W_{f}(\nu, \tau)\right|^{2} \mathrm{~d} \tau \int_{t_{1}}^{t_{2}}\left|W_{g}(\nu, \tau)\right|^{2} \mathrm{~d} \tau}}$.

The absolute value of the complex function $C_{f g}(\nu)$ belongs to the interval $[0,1]$ and characterizes the level of correlation of oscillations at the frequency $\nu$. The phase

$\phi(\nu)=\tan ^{-1}\left(\frac{\mathfrak{I}\left[C_{f g}(\nu)\right]}{\mathfrak{R}\left[C_{f g}(\nu)\right]}\right)$

shows the mean phase difference of the corresponding pulsations.

As the real experimental data are nonperiodic and nonstationary, the boundaries and significant peculiarities of the data influence the spectrum of the data and input in the spectral energy. To avoid this, we make an offset from the boundaries, depending on the frequency under consideration, which increases 


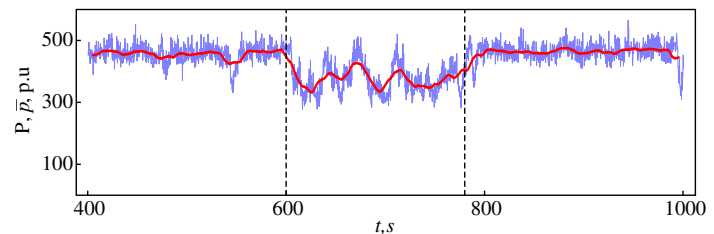

(a)

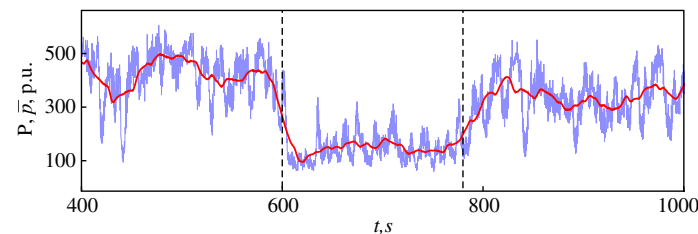

(b)

Fig. 2 (a, b) Original $P$ (blue thin line) and smoothed $\bar{p}$ (red thick line) LDF records for two subjects during the CPT. Vertical dashed lines indicate the time interval of cooling. The smoothing is done by a moving average with a 20-s window.

Table 1 Average relative variation in skin perfusion $\delta P_{i j}$ for three time intervals: before $(i=1)$, during $(i=2)$, and after $(i=3)$ the cooling.

\begin{tabular}{lcc} 
Value & Median & Mean \\
\hline$\delta P_{1,2}$ & $0.55 \pm 0.11$ & $0.45 \pm 0.22$ \\
$\delta P_{3,2}$ & $0.46 \pm 0.14$ & $0.45 \pm 0.19$ \\
$\delta P_{1,3}$ & $0.07 \pm 0.08$ & $0.08 \pm 0.09$ \\
\hline
\end{tabular}

when frequency decreases. Low-frequency pulsations $(0.01$ to $0.02 \mathrm{~Hz}$ ) associated with the endothelial activity were not taken into account as the characteristic time of the endothelial activity was close to the duration of the functional test (180 s), which leads to insufficient statistic.

All the original algorithms for the spectral analysis and the statistical analysis of the data were implemented in Mathematica 8.0.

Table 2 Spearman correlation of $\langle P\rangle$ and SD of pulsations $p_{k}$ for three time intervals and four frequency bands, calculated over all subjects.

\begin{tabular}{lccc} 
Frequency band & Before & During cooling & After \\
\hline Cardiac & 0.67 & 0.27 & 0.019 \\
Respiratory & 0.68 & 0.20 & -0.083 \\
Myogenic & 0.19 & 0.31 & -0.007 \\
Neurogenic & 0.14 & 0.12 & -0.068 \\
\hline
\end{tabular}
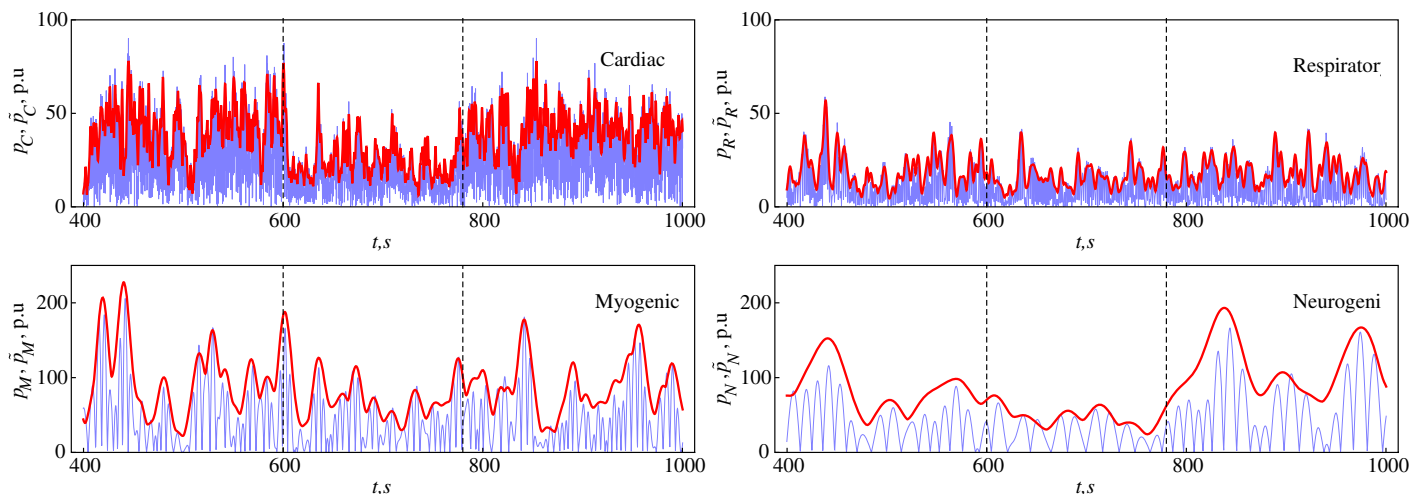

Fig. 3 Results of IWT (blue thin lines) and WCE (red thick lines) filtering of the signal, shown in Fig. 2(a) for the cardiac, respiratory, myogenic, and neurogenic frequency bands. Time limits of the cooling are marked by vertical dashed lines.

\section{Results}

\subsection{Dynamics of Average Laser Doppler Flowmetry Signal}

Two typical CPT records of tissue perfusion $P$ on the distal phalange of the palm surface of the fingers are presented in Fig. 2. In both cases, the cooling induces a stepwise decrease in perfusion on the contralateral limb, which recovers after finishing the cooling. The first example [shown in Fig. 2(a)] demonstrates a rather strong reaction of the perfusion to the cooling. From an initial level of about 450 p.u., the perfusion went down during the cooling to a level of about 100 p.u. The second example [Fig. 2(b)] shows a more quiet behavior from the cooling time and a weaker reaction of the perfusion (about 450 p.u. before and about 350 p.u. during the cooling). To quantify the response of perfusion to the CPT we used relative variation of the LDF signal defined as $\delta P_{i j}=\frac{\left\langle P_{i}\right\rangle-\left\langle P_{j}\right\rangle}{\left\langle P_{i}\right\rangle}$, where indexes $i, j=1,2,3$ indicate time interval. ${ }^{32}$ Table 1 shows the averaged over the whole group values of $\delta P_{i j}$. One can see that the perfusion decreases during the cooling in all 14 samples and the median relative variation is $\delta P_{12}=0.55 \pm 0.11$. Thus, all the subjects in the group demonstrated a similar reaction of the mean perfusion to the CPT, despite the individual features in the recorded signals.

\subsection{Laser Doppler Flowmetry Pulsations}

As similar reaction of average perfusion does not imply a similar reaction in the oscillations. In the example shown in Fig. 2(a), cooling provoked a decrease in the amplitude of the perfusion pulsations, which is contradictory to another example presented 


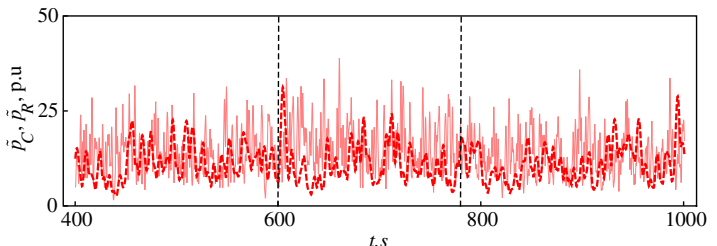

(a)

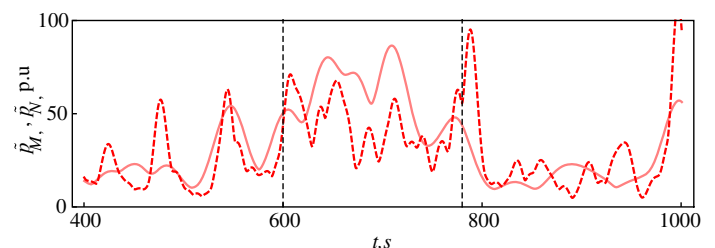

(b)

Fig. 4 Results of WCE filtering of the signal, shown in Fig. 2(b). (a) The cardiac (solid) and respiratory (dashed); (b) myogenic (solid) and neurogenic (dashed) frequency bands.

in Fig. 2(b). Thereby, the oscillations demonstrate more complicated behavior during CPT with significant individual differences. To study the pulsations in detail, we made the wavelet filtration and separate oscillations in four frequency bands of interest: cardiac $p_{C}(t)$, respiratory $p_{R}(t)$, myogenic $p_{M}(t)$, and neurogenic $p_{N}(t)$.
First, we aimed to answer the question whether the intensity of oscillations in a given frequency band is related to the mean perfusion. For this purpose, we calculated the SD of pulsations $p_{k}$ in each frequency band for each subject and estimated the Spearman correlation of $\langle P\rangle$ and SD of pulsation in a given frequency band over all the subjects (Table 2).
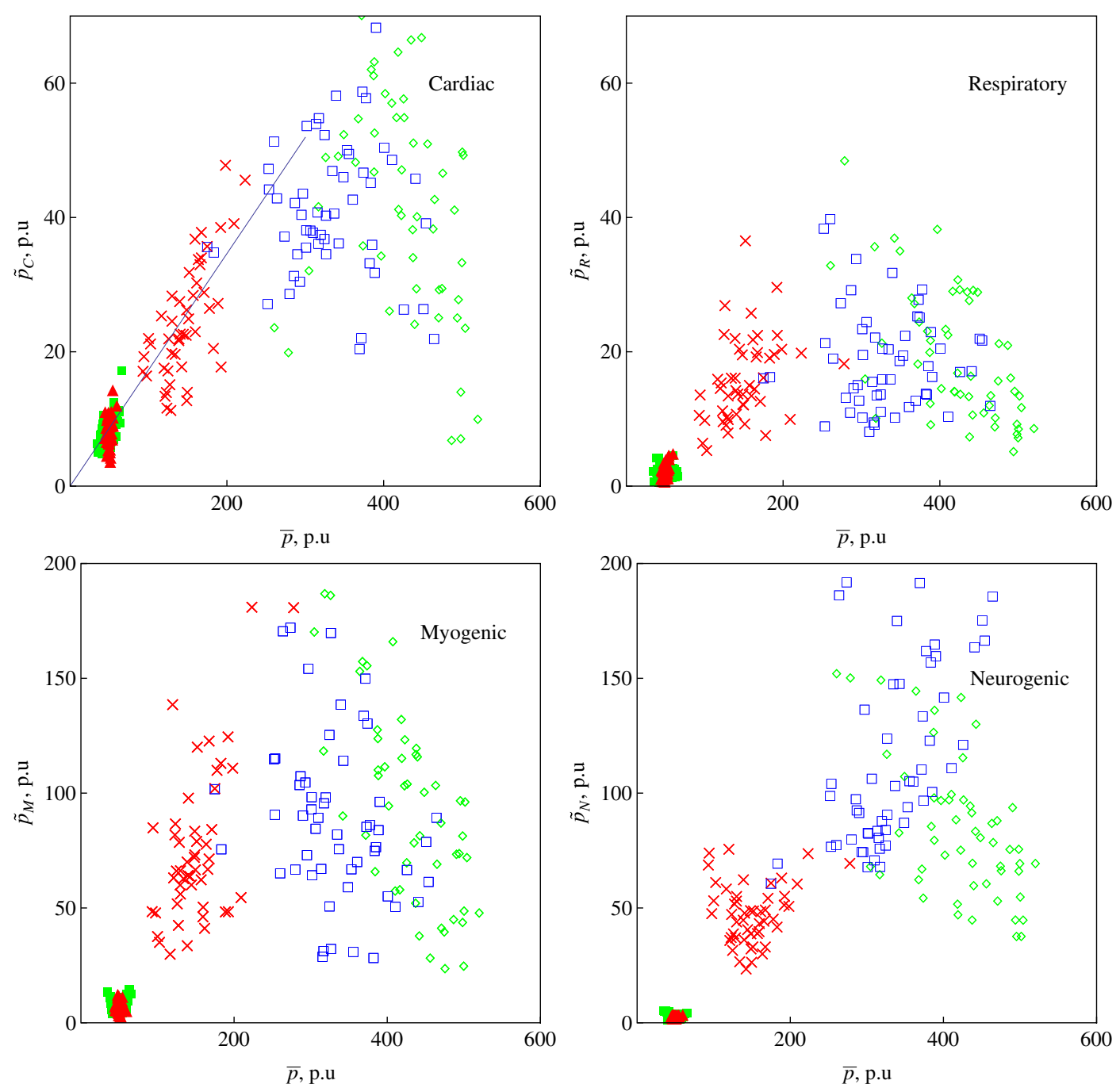

Fig. 5 Scatter diagram of $\bar{p}$ and $\tilde{p}$ for the example presented in Fig. 2(a). Green open diamonds corresponds to data collected from the distal phalanx in the rest period before cooling, red crosses correspond to the time of cooling, while the blue open rectangles were obtained after cooling. Data collected from the palm are indicated by closed symbols: green rectangles before, red triangles during, and blue circles after cooling. All points for the perfusion less than 300 p.u. are fitted by the linear law. Different panels corresponds to different frequency bands: (a) cardiac, (b) respiratory, (c) myogenic, and (d) neurogenic. 
Table 3 Correlation of variations in $\bar{p}$ and $\tilde{p}_{k}$ for example presented in Fig. 2(a) for three time intervals.

\begin{tabular}{lccc} 
Frequency band & Before & During cooling & After \\
\hline Cardiac & -0.59 & 0.77 & -0.47 \\
Respiratory & -0.3 & 0.49 & -0.26 \\
Myogenic & -0.68 & 0.31 & -0.55 \\
Neurogenic & -0.45 & 0.12 & -0.27 \\
\hline
\end{tabular}

A significant correlation is observed only in the frequency bands associated with passive mechanisms of vascular tone regulation (cardiac and respiratory) in the basal conditions. This means that under relaxed conditions, the oscillations of blood flow in the microvascular bed caused by the passive mechanisms are slightly connected with the mean value of perfusion and this value is at the boundary of significance, while cooling practically destroys correlation. The amplitudes of the pulsations corresponding to the active mechanisms of blood flow regulation are fully independent from the mean value of blood flow.

\subsection{Temporal Analysis of Laser Doppler Flowmetry Signal}

Next, we studied the behavior of both components of perfusion, namely, the average $\bar{p}(t)$ and pulsatile $p_{k}(t)$ components for individuals. The pulsations $p_{k}(t)$ were calculated by filtration of the LDF signals in a defined frequency band using the IWT Eq. (3). Figure 3 presents an example of the dynamics of the absolute values of the filtered signal $p_{k}(t)$ (blue thin lines), together with the envelope $\tilde{p}_{k}(t)$ calculated by the WCE algorithm Eq. (6) (red thick lines in Fig. 3). Indeed, WCE technique gives an envelope for the filtered signal and indicates the variations of the amplitude of the pulsations in a given frequency band. Below, we consider only the result of the WCE technique and indicate the amplitude variations of the oscillations by $\tilde{p}_{C}(t)$ for the cardiac, $\tilde{p}_{R}(t)$ for respiratory, $\tilde{p}_{M}(t)$ for myogenic, and $\tilde{p}_{N}(t)$ for neurogenic components.

Figure 3 shows that cooling provokes different variations in the amplitude of the pulsations in different frequency bands (e.g., the pulsations associated with respiration do not react at all). The amplitude of the oscillation associated with the cardiac activity reduces during cooling, where a somewhat weaker reaction is observed in the myogenic and neurogenic frequency ranges.

Figure 4 presents the result of wavelet filtration of the LDF signal, shown in Fig. 2(b), which differs from the first signals [Fig. 2(a)], due to much weaker reaction in average perfusion and the increasing SD of the pulsations. Figure 4 shows that the reaction of this subject in the separated frequency bands also differs from the reaction of the first subject. So, $\tilde{p}_{M}$ and $\tilde{p}_{N}$ essentially increase during the cooling [Fig. 4(b)], while the reaction in the cardiac and respiratory ranges is not pronounced.

To clarify the relationship of $\bar{p}$ and $\tilde{p}$, in Fig. 5, we plotted the scatter diagram for all four frequency bands under consideration [again, for the signals shown in Fig. 2(a)]. These scatter diagrams demonstrate the complicated nonlinear relationship of $\bar{p}$ and $\tilde{p}_{k}$ in all four frequency bands. The clearest dependency is seen in the cardiac frequency band [Fig. 5(a)]. For low perfusion (less than half the maximal), the amplitude of oscillations is proportional to the perfusion. At high perfusion, the amplitude of oscillations decreases with increasing perfusion, providing an inverse relation of $\bar{p}$ and $\tilde{p}$. The similar relationship of the oscillating and average component is observed for lower frequency pulsations [Figs. 5(b)-5(d)], but the points show a higher divergence, which is explained by the limited statistics. In terms of linear correlation, this means that the Pearson correlation coefficients change the sign (Table 3). For low and high values of perfusion, $\bar{p}$ and $\tilde{p}$ are clearly related, but they seem to be independent at moderate values of perfusion. Thereby, the amplitudes of the pulsations $\tilde{p}_{k}$ are related to the average perfusion $\bar{p}$ in a complex nonlinear way.

Strong anticorrelation of two variables means that if one signal decreases the other one increases, and vice versa. In other words, for the oscillatory process, it means that the oscillations are in the opposite phase. The wavelet frequency-by-frequency cross-correlation of $\bar{p}$ and the pulsatile components $\tilde{p}_{k}$ was calculated to study this. In Fig. 6, the modulus and the phase of the wavelet cross-correlation function Eq. (7) for $\bar{p}$ and $\tilde{p}_{C}$ (solid lines) and for $\bar{p}$ and $\tilde{p}_{R}$ (dashed lines) for the whole range of modulation frequency $(0.01$ to $1 \mathrm{~Hz})$ is demonstrated. The blue lines in Fig. 6 correspond to cross-correlations for the subject at rest, while the red lines show the correlations during the cooling.

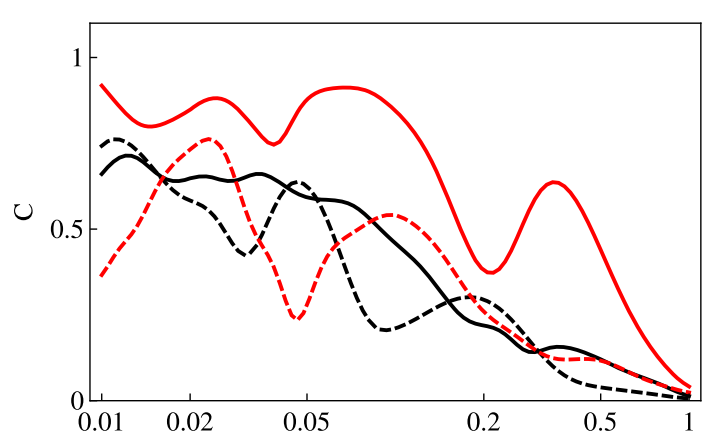

(a)

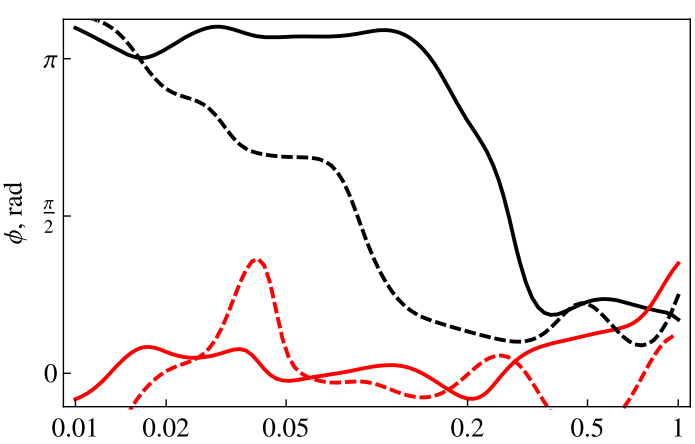

(b)

Fig. 6 (a) Modulus and (b) phase shift of wavelet cross-correlation function for $\bar{p}$ and $\tilde{p}_{k}$. Solid lines indicate cross-correlation coefficients for $\bar{p}$ and $\tilde{p}_{C}$, dashed for $\bar{p}$ and $\tilde{p}_{R}$, blue lines for the rest, red lines for cooling. 


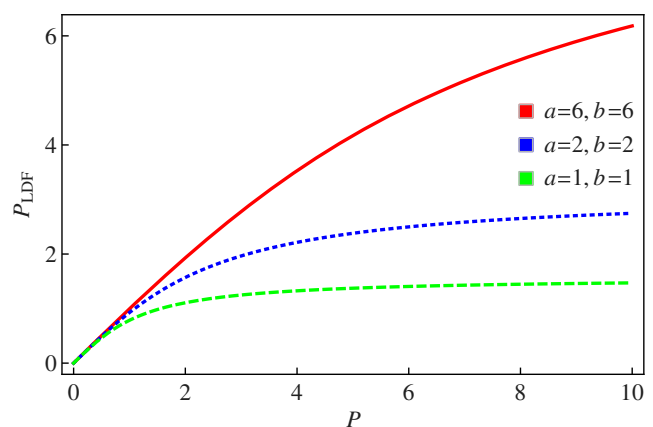

(a)

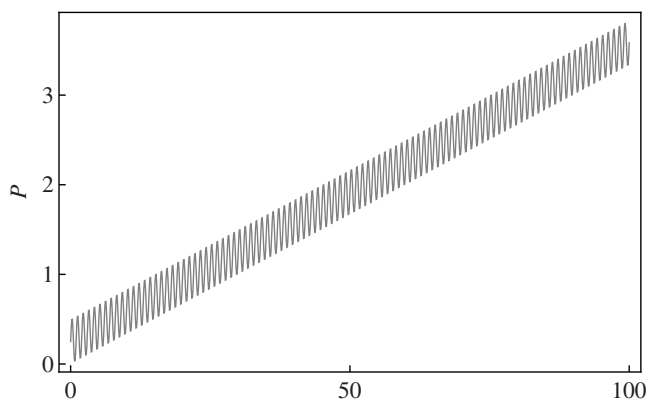

(b)

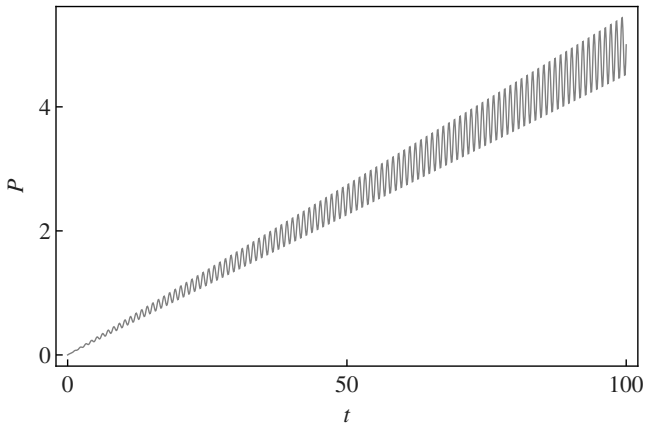

(d)

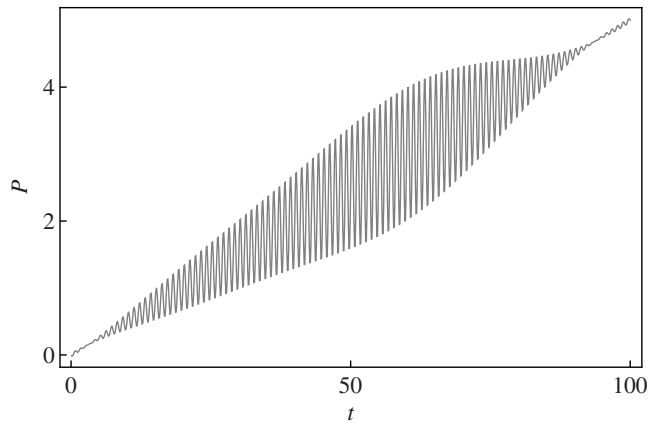

(f)

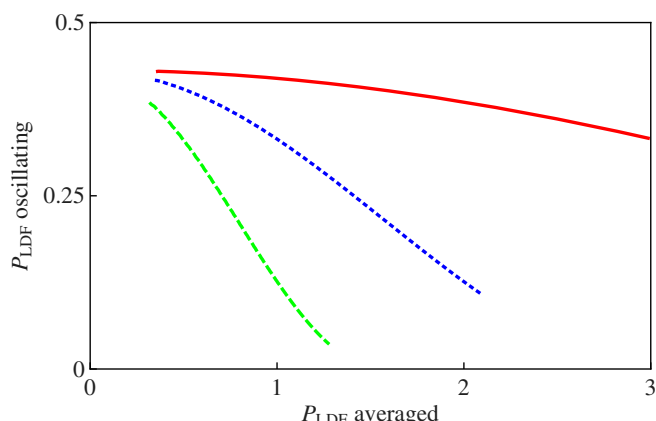

(c)

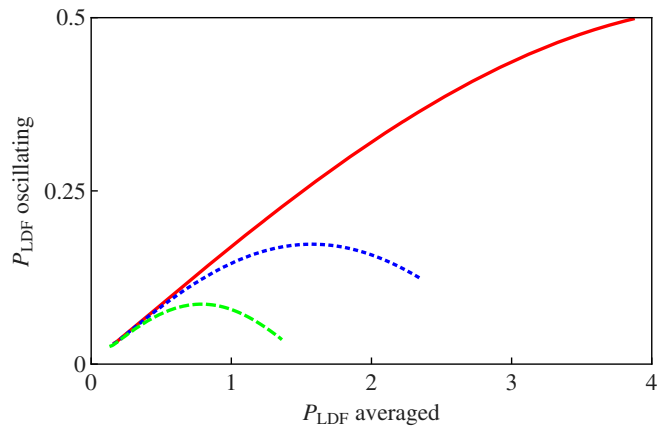

(e)

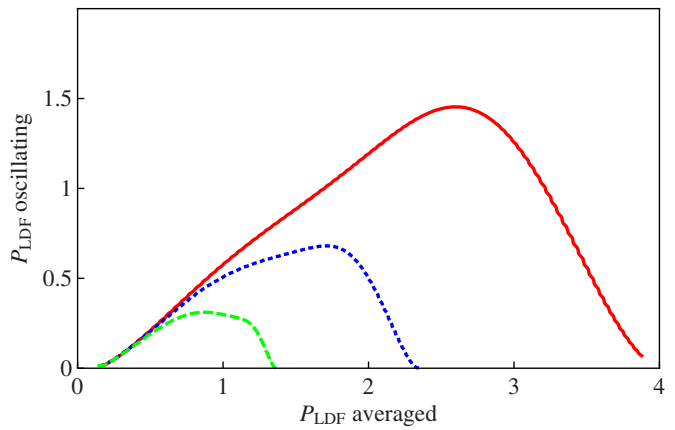

(g)

Fig. 7 Artificial examples of the behavior of perfusion and the result of observation with different kinds of transform functions. In panel (a), a variety of transform functions $G(P)=a \arctan (P / b)$ for different values of parameters $a$ and $b$ are presented. In the second, third, and fourth lines, we consider the effect of the transfer function on perfusion: oscillation of perfusion does not depend from the averaged value (b), amplitude of perfusion pulsation is proportional to its averaged value (d), perfusion increases with a nonlinear shape of amplitude of oscillations (f). In the right column (c), (e), (g) $\tilde{P}_{\text {LDF }}-\bar{P}_{\text {LDF }}$ diagrams for corresponding examples are presented. 
The highest correlation is observed for the cardiac signal during the cooling [this state corresponds to the left linear domain in Fig. 5(a)]. The modulus of correlation in the respiratory frequency band is moderate and cooling does not induce significant changes in its value. Changes in the phase shift of $\bar{p}$ and $\tilde{p}$, shown in Fig. 6(b), suggest dramatic changes in the relationship of the two components under consideration. At rest (blue line), the phase shift of the low-frequency pulsations of $\bar{p}$ and $\tilde{p}_{C}$ is close to $\pi$ for $0.01<\nu<0.1 \mathrm{~Hz}$ and tends to zero during the cooling (red line). For the phase of cross-correlation for $\bar{p}$ and $\tilde{p}_{R}$, the anticorrelation at rest is less pronounced (the phase shift is close to $\pi$ at $\nu \approx 0.01$ and decreases for higher frequencies, but the tendency is similar). Under cooling, both signals $\tilde{p}_{C}$ and $\tilde{p}_{R}$ demonstrate a direct correlation with the average perfusion (no phase shift).

\subsection{Nonlinear Transfer Function}

Analyzing and interpreting the results obtained, one should take into account the LDF transfer function $P_{\mathrm{LDF}}=G(P)$, which relates the measured perfusion $P_{\mathrm{LDF}}$ to the real tissue perfusion $P$ and is actually nonlinear. The function $G(P)$ depends on the optical properties and peculiarities of the morphological structure of the tissue of a given subject and varies for individuals. The detailed form of this function is unknown and we will illustrate its possible influence basing on general framework of LDF technique. ${ }^{16}$

Several models of dependence of the perfusion oscillation amplitude on mean perfusion and transfer function are considered. According to Ref. 16, it is assumed that the transfer function $G(P)$ is linear for low $P$ and then saturates at large $P$. We model it as $G(P)=a \arctan (P / b)$ and consider weak (red), moderate (blue), and strong (green) saturation [Fig. 7(a)]. Then, three test variations of real perfusion in time $P(t)$ are taken: increasing perfusion with constant amplitude of oscillations [Fig. 7(b)]; increasing perfusion with linear increasing amplitude of oscillations [Fig. 7(d)]; increasing perfusion with a nonlinear shape of amplitude of oscillations [Fig. 7(f)]. Using the transfer function, $P_{\mathrm{LDF}}$ signal is calculated, and then it is decomposed on the averaged and oscillating parts [Figs. 7(c), $7(\mathrm{e})$, and $7(\mathrm{~g})]$.

Thus, a $\tilde{P}-\bar{P}$ diagram with a descending branch can appear under applied transfer function for the case of perfusion oscillations, which monotonically increase with perfusion. If oscillations decrease at high perfusion for some intrinsic reason
$\tilde{P}-\bar{P}$ diagram becomes more complicate with strong asymmetry of ascending and descending branches [Figs. 7(f) and 7(g)]. Example, shown in the Fig. 7(f) by green and blue lines, exhibit three parts in $\tilde{P}-\bar{P}$ diagram: under low perfusion, the curve is defined by the linear dependence of $\tilde{P}$ on $\bar{P}$; at moderate perfusion, the transfer function plays the major role and decreases the slope of the curve; and at high perfusion, the decay of oscillations is provided by some intrinsic reason.

These simple test examples demonstrate that the transfer function strongly distorts $\tilde{P}-\bar{P}$ diagram that is why, especially for high perfusion, the interpretation should be done with high accuracy.

\subsection{Group Statistics}

All the previous results were presented for a couple of examples and so the aim of this section is to find some statistical characteristics for the whole group of subjects and to clarify whether the observed behavior of LDF oscillations is typical for all subjects. Correlation coefficients of $\bar{p}$ and $\tilde{p}_{k}$, which were shown for one subject in Table 3, were calculated for all the subjects for three time intervals. The results obtained are summarized in a Box-Whisker diagram in Fig. 8(a). In the majority of measurements, we observe that cooling causes the change of the sign of the correlation coefficients from negative to positive. Thus, the microvascular reaction described above for one subject is typical.

\subsection{Dorsal Part of the Palm}

In five subjects from the whole group, we collected simultaneous data on the fingertip and on the dorsal part of the palm between the base of the thumb and the index finger. This part of the palm has nonglabrous skin with a significantly smaller density of capillaries and perfusion is not high (up to 30 p.u. in the example under consideration), see Fig. 2(a). Data from the dorsal part were included in the scatter diagrams for $\bar{p}$ and $\tilde{p}_{k}$ (Fig. 5) and indicated by closed symbols. Remarkably, the dependence of $\tilde{p}_{C}$ from $\bar{p}$ obeys the same linear law as for the fingertip and for dorsal part. So, the relation of $\bar{p}$ and $\tilde{p}_{C}$ does not depend on the point of data collecting but has individual differences. Correlation coefficients for these five records are shown in the Box-Whisker diagram [Fig. 8(b)]. Linear correlation coefficients are positive on the dorsal part of the hand and slightly decreases because of the contralateral cooling.

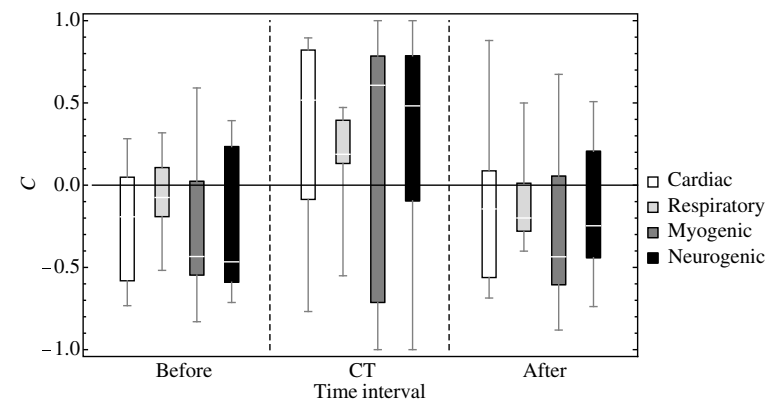

(a)

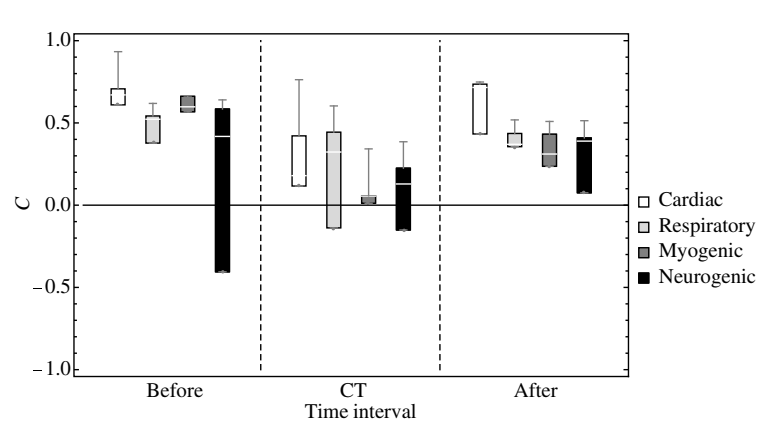

(b)

Fig. 8 Statistics over the whole group of subjects ( 14 for the distal phalanges and 5 for the dorsal side of the arm) for the correlation coefficient for $\bar{p}(t)$ and $\tilde{p}_{k}(t)(k=C, R, N, M)$, presented as Box-Whisker diagrams. In (a) data are recorded from distal phalanges (14 records), while the (b) shows from the dorsal part of the hand (five records). 


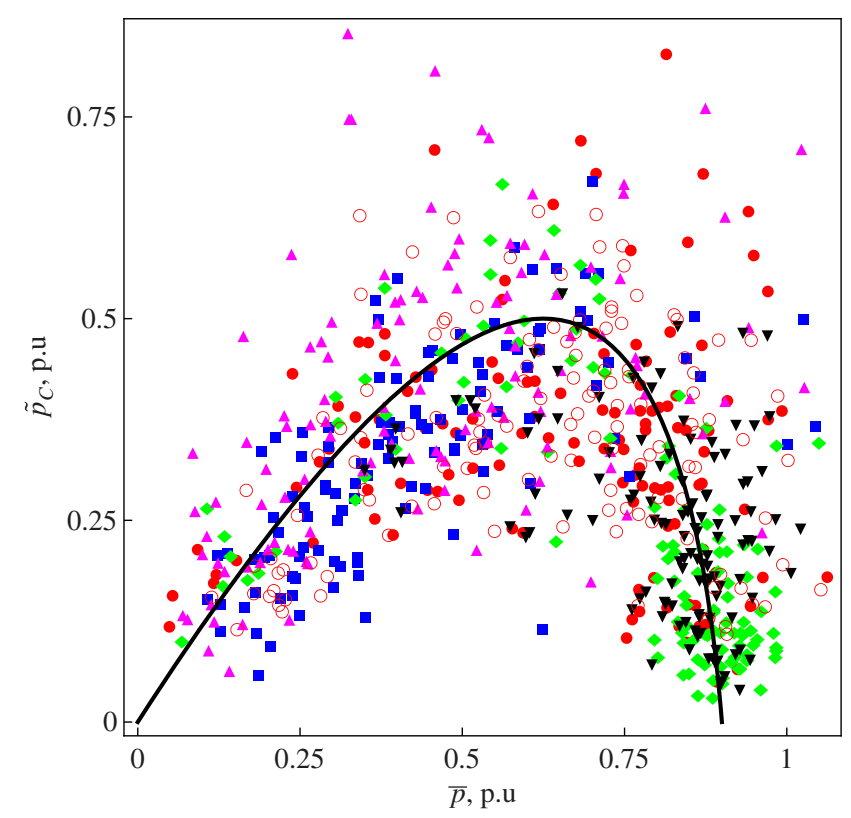

Fig. 9 Scatter diagram for six individuals to illustrate the relation of $\bar{p}^{\prime}$ and $\tilde{p}^{\prime}$. Different symbols indicate different subjects.

\section{Discussion and Conclusion}

The relationship between average and oscillating perfusion, measured by means of LDF, was studied during the contralateral CPT. The LDF signal was recorded on two different sites of the hand: dorsal part of the arm and finger pad. Such protocol makes it possible to get a wide range of perfusion. The average perfusion always decreases during cooling, while the oscillating component demonstrates differently directed reaction.

This behavior became clear when the intrinsic nonlinear relation of the average $\bar{p}(t)$ and oscillatory components $[\tilde{p}(t)]$ were established. For low perfusion (up to half of the maximal value), $\tilde{p}(t)$ is proportional to $\bar{p}(t)$, whereas with saturation of the tissue by blood, the oscillating component becomes weaker and converges to zero at high perfusion. This relation manifests itself as a positive correlation of $\bar{p}(t)$ and $\tilde{p}(t)$ under moderate perfusion and as an anticorrelation for high perfusion.

In the majority of measurements, the perfusion of the acral skin was high, more than 300 p.u., and weak pulsations were observed. These weak oscillations can be explained by the large contribution of capillary blood flow into the LDF signal. The blood flow in the capillaries is stationary with a low-oscillating component, ${ }^{33}$ so oscillations in the resulting LDF signal become low.

The reduction of perfusion in the fingertip and on the surface of the dorsal part of the palm was provided in our experiments by cooling the contralateral limb. Such cooling induces activation of the sympathetic nervous system, which results in cutaneous vasoconstriction and a decrease in skin blood flow. This causes the redistribution of blood flow through elements of the microcirculation system, namely, arteriolar vasoconstriction, a high tone of precapillary sphincters, and blood flow through the arteriovenous anastomoses. ${ }^{15}$ In this case, the LDF probe collects signal mostly from the arterioles, which have a muscular wall and demonstrate pulsations. The arteriolar tone pulsations are correlated with the perfusion.

In the dorsal part of the arm, the perfusion is lower than that in the finger pad because of the morphological difference of the skin and different capillary density. Cooling causes a decrease of perfusion but the relation of $\bar{p}(t)$ and $\tilde{p}_{C}(t)$ did not vary.
Remarkably, $\bar{p}(t)$ and $\tilde{p}_{C}(t)$ data collected from the dorsal part of the hand obey the same linear law as data from the fingertip under low perfusion state.

The range of LDF signal variations differs from subject to subject, but the relation of $\bar{p}(t)$ and $\tilde{p}(t)$ demonstrates common behavior. To assemble all measurements we used, the dimensionless values $\bar{p}^{\prime}(t)$ and $\tilde{p}^{\prime}(t)$, which were calculated in two steps. First, the angle coefficient $\theta$ for the linear part of this curve was found and then all data were normalized to $\theta=1$. Second, the perfusion for a given subject was normalized to the maximum perfusion: $\bar{p}^{\prime}(t)=\bar{p}(t) / \max (P)$ and $\tilde{p}^{\prime}(t)=\tilde{p}(t) / \max (P)$. Results of this two-step procedure are shown in Fig. 9, which explains the contradirectional reaction of the pulsatile component of the LDF signal on the contralateral cooling. We conclude that the response of the oscillating component to the CPT depends on the initial value of perfusion and degree of vasoconstriction. In the relaxed conditions, the perfusion is moderate and the contralateral cooling provokes vasoconstriction and reduces the oscillating component. If the initial perfusion is strong, the oscillating component is weak and contralateral cooling provokes decrease of perfusion and increase of $\tilde{p}$.

Respiratory $\tilde{p}_{R}$, myogenic $\tilde{p}_{M}$, and neurogenic $\tilde{p}_{N}$ pulsations behave themselves similar to the cardiac ones $\tilde{p}_{C}$. While perfusion is moderate, the amplitude of pulsations is proportional to $\bar{p}$; and with the increase of $P$, pulsations become less intensive, the divergence of the points is rather large for low-frequency oscillation.

Thus, the oscillating component of LDF signal is correlated with its average in a complicated way. Wavelet correlation analysis demonstrated that for low perfusion, the variations of both components are inphase, whereas in the saturated stage they are in contraphase. Discussing this nontrivial behavior, one should take into account that when measured by means of LDF device perfusion, $P_{\mathrm{LDF}}$ is nonlinearly related to the real perfusion. A saturation of the LDF signal appears under high RBC concentration, which often exists in the finger pad in the relaxed state. We illustrated by simple tests that both effects, namely, the "real physiological" dependence of amplitude of pulsations on the averaged perfusion and the nonlinear transfer function, can contribute to the observed relation.

The negative correlation of $\bar{p}(t)$ and $\tilde{p}(t)$ for individuals under basal conditions does not imply a similar intragroup correlation. The nonlinear curve of $\bar{p}(t)$ and $\tilde{p}(t)$ looks similar, but varies from subject to subject, namely each has an own scale factor. Mean over time values of $\bar{p}(t)$ and $\tilde{p}(t)$ for the intragroup statistics are positively correlated.

Recently, the analysis of blood flow oscillation has been used to detect the abnormality of the microcirculation system in variety of pathologies such as diabetic mellitus, ${ }^{34}$ melanoma, ${ }^{35}$ Rheino syndrome, and peripheral arterial obliterative disease. ${ }^{36}$ The present work is devoted to methodological aspects of LDF measurements under functional tests and gives rise to research to widen our knowledge on the functional state of the microcirculation system.

\section{Acknowledgments}

This work was supported by the Russian Science Foundation under Project No. 14-15-00809. The authors kindly thank A. Tankanag and A. Dunaev for their useful discussion and anonymous referees for careful and insightful reports on the submitted version of the paper, which resulted in necessary improvements. 


\section{References}

1. A. Stefanovska, M. Bracic, and H. D. Kvernmo, "Wavelet analysis of oscillations in the peripheral blood circulation measured by laser Doppler technique," IEEE Trans. Biomed. Eng. 46, 1230-1239 (1999).

2. M. Rossi et al., "Skin vasomotion investigation: a useful tool for clinical evaluation of microvascular endothelial function?," Biomed. Pharmacother. 62(8), 541-545 (2008).

3. R. Martini, V. Ticcinelli, and A. Bagno, "Changes of the cutaneous flowmotion pattern after limb revascularization in patients with critical ischemia," Clin. Hemorheol. Microcirc. 56(4), 347-358 (2014).

4. A. Krupatkin, "Dynamic oscillatory circuit of regulation of capillary hemodynamics," Hum. Physiol. 33(5), 595-602 (2007).

5. C. J. Abularrage et al., "Evaluation of the microcirculation in vascular disease," J. Vasc. Surg. 42(3), 574-581 (2005).

6. M. Stern, "In vivo evaluation of microcirculation by coherent light scattering," Nature 254(5495), 56-58 (1975).

7. A. Stefanovska, "Physics of the human cardiovascular system," Contemp. Phys. 40(1), 31-55 (1999).

8. J. Schmidt et al., "Induced periodic hemodynamics in skeletal muscle of anesthetized rabbits, studied with multiple laser Doppler flow probes," Int. J. Microcirc. 15(1), 28-36 (1995).

9. N. Charkoudian, "Mechanisms and modifiers of reflex induced cutaneous vasodilation and vasoconstriction in humans," J. Appl. Physiol. 109(4), 1221-1228 (2010).

10. H. Daanen, "Finger cold-induced vasodilation: a review," Eur. J. Appl. Physiol. 89(5), 411-426 (2003).

11. Y. Isii et al., "Ice-water hand immersion causes a reflex decrease in skin temperature in the contralateral hand," J. Physiol. Sci 57(4), 241-248 (2007).

12. S. Frank and S. Raja, "Reflex cutaneous vasoconstriction during cold pressor test is mediated through a-adrenoceptors," Clin. Auton. Res. 4(5), 257-261 (1994).

13. I. Mizeva, P. Frick, and S. Podtaev, "Skin blood flow and temperature oscillations during cold pressor test," in 37th Annual Int. Conf. of the IEEE Engineering in Medicine and Biology Society (EMBC'15), pp. 7382-7385 (2015).

14. L. W. Sheppard et al., "Oscillatory dynamics of vasoconstriction and vasodilation identified by time-localized phase coherence," Phys. Med. Biol. 56(12), 3583 (2011).

15. J. Sonksen and J. Craggs, "Circulation of the skin," Curr. Anaesthesia Crit. Care 10(2), 58-63 (1999).

16. I. Fredriksson, C. Fors, and J. Johansson, "Laser Doppler flowmetry - a theoretical framework," Department of Biomedical Engineering, Linkoping University, www.imt.liu.se/bit/ldf/ldfmain.html (2007).

17. I. Fredriksson, M. Larsson, and T. Stroemberg, "Model-based quantification of skin microcirculatory perfusion," Chapter 14 in Computational Biophysics of the Skin, B. Querlex, Ed., pp. 395-418, Pan Stanford Publishing (2014).

18. A. Obeid, "In vitro comparison of different signal processing algorithms used in laser Doppler flowmetry," Med. Biol. Eng. Comput. 31, 43-52 (1993).

19. A. Liebert, M. Leahy, and R. Maniewski, "Multichannel laser-Doppler probe for blood perfusion measurements with depth discrimination multichannel laser-Doppler probe for blood perfusion measurements with depth discrimination," Med. Biol. Eng. Comput. 36, 740-747 (1998).

20. A. Liebert et al., "Optoelectronic standardization of laser Doppler perfusion monitors," Rev. Sci. Instrum. 70, 1352-1354 (1999).

21. D. Lapitan and D. Rogatkin, "Variable hyperemia of biological tissue as a noise source in the input optical signal of a medical laser Doppler flowmeter," J. Opt. Technol. 83(1), 36-42 (2016).
22. C. Aalkjer, D. Boedtkjer, and V. Matchkov, "Vasomotion-what is currently thought?," Acta Physiol. 202(3), 253-269 (2011).

23. P. Nicoll and R. Webb, "Vascular patterns and active vasomotion as determiners of flow through minute vessels," Angiology 6(4), 291-310 (1955).

24. R. Gniadecki, M. Gniadecka, and J. Serup, Examination of Periodic Fluctuations in Cutaneous Blood Flow, 2nd ed., Academic Press (2006).

25. W. Lovallo, "The cold pressor test and autonomic function: a review and integration," Psychophysiology 12(3), 268-282 (1975).

26. T. Lewis, "Observations upon the reactions of the vessels of the human skin to cold," Heart 15, 177-208 (1930).

27. A. Kistler, C. Mariauzouls, and K. Von Berlepsch, "Fingertip temperature as an indicator for sympathetic responses," Int. J. Psychophysiol. 29(1), 35-41 (1998).

28. I. Sendowski et al., "Sympathetic stimulation induced by hand cooling alters cold-induced vasodilatation in humans," Eur. J. Appl. Physiol. 81(4), 303-309 (2000).

29. S. Mallat, A Wavelet Tour of Signal Processing: The Sparse Way, 3rd ed., Academic Press (2008).

30. S. Podtaev et al., "Wavelet-analysis of skin temperature oscillations during local heating for revealing endothelial dysfunction," Microvasc. Res. 97(0), 109-114 (2015).

31. P. Frick, I. Mizeva, and S. Podtaev, "Skin temperature variations as a tracer of microvessel tone," Biomed. Signal Process. Control 21, 1-7 (2015).

32. E. Smirnova et al., "Assessment of endothelial dysfunction in patients with impaired glucose tolerance during a cold pressor test," Diabetes Vasc. Dis. Res. 10(6), 489-497 (2013).

33. A. Guyton and J. Hall, Textbook of Medical Physiology, Guyton Physiology Series, Elsevier Saunders (2006).

34. J. D. Lefrand et al., "Sympathetic mediated vasomotion and skin capillary permeability in diabetic patients with peripheral neuropathy," Diabetologia 46, 40-47 (2003).

35. G. Lancaster et al., "Dynamic markers based on blood perfusion fluctuations for selecting skin melanocytic lesions for biopsy," Sci. Rep. 5, 12825 (2015).

36. M. Rossi and A. Carpi, "Skin microcirculation in peripheral obliterative disease," Biomed. Pharmacother. 58, 427-431 (2004).

Irina Mizeva graduated from Perm State University in 2003, where she also received her PhD in mechanics in 2008. In 2012, she joined the Biomedical Group led by Prof. P. Frick and Dr. S. Podtaev at the Institute of Continuous Media Mechanics, where she currently holds a research position. Her research interests include signal processing, wavelet analysis, the experimental study of the human microcirculation system, and magnetohydrodynamic turbulence.

Peter Frick received his diploma degree in physics from the University of Perm in 1974, and his PhD in physics and mathematics in 1981, and his doctor of sciences degree in 1991. Since 1992, he has been the head of the Laboratory of Physical Hydrodynamic, Institute of Continuous Media Mechanics, Perm, Russia, and a full professor at Perm State University. His research interests include turbulence, convection, magneto-hydrodynamics, and wavelet analysis.

Sergey Podtaev works at Control Systems Ltd., a Russian company that specializes in the manufacture of electronic equipment for industry. He is the head of the Department of New Developments and Innovations. In 2007, he started to work on the development of new approaches in medical diagnostics. His areas of expertise include high-precision temperature measurements, low-amplitude skin temperature, and microvascular dysfunction. 